\title{
THE MAX-MIN-MIN PRINCIPLE OF PRODUCT DIFFERENTIATION
}

\author{
Asim Ansari \\ Graduate School of Business, Columbia University, New York, NY, U.S.A., \\ E-mail:aansari@research.gsb.columbia.edu.
}

\section{Nicholas Economides}

Stern School of Business, New York University, New York, NY, U.S.A., E-mail:neconomi@stern.nyu.edu.

\section{Joel Steckel}

Stern School of Business, New York University, New York, NY, U.S.A., E-mail:jsteckel@stern.nyu.edu.

\begin{abstract}
We analyze two and three-dimensional variants of Hotelling's model of differentiated products. In our setup, consumers can place different importances on each product attribute; these are measured by weights on the disutility of distance in each dimension. Two firms play a two-stage game; they choose locations in stage 1 and prices in stage 2. We seek subgame-perfect equilibria. We find that all such equilibria have maximal differentiation in one dimension only; in all other dimensions they have minimum differentiation. An equilibrium with maximal differentiation in a certain dimension occurs when consumers place sufficient importance (weight) on that attribute. Thus, depending on the importance consumers place on each attribute, in two dimensions there is a max-min equilibrium, a min-max equilibrium, or both. In three dimensions, depending on the weights, there can be a max-min-min equilibrium, a min-max-min equilibrium, a min-min-max equilibrium, any two of these, or all three.
\end{abstract}

\section{INTRODUCTION}

A primary goal of the theory of product differentiation is the determination of market structure and conduct of firms that can choose the specifications of their products besides choosing output and price. Traditional models of product differentiation and marketing have focused on products that are defined by one characteristic only (see Hotelling, 1929; Vickrey, 1964; D’Aspremont, Gabszewicz, and Thisse, 1979; Salop, 1979; Economides, 1984; Anderson, de Palma, and Thisse, 1922; among others in economics and Hauser and Shugan, 1983; Moorthy, 1988; and Kumar and Sudarshan, 1988, in marketing). One-characteristic models are sufficient for understanding the interaction between product specification and price. The main question in this setting is the degree of product

(C) Blackwell Publishers 1998.

Blackwell Publishers, 350 Main Street, Malden, MA 02148, USA and 108 Cowley Road, Oxford, OX4 1JF, UK. 
differentiation at equilibrium-does the acclaimed "Principle of Minimum Differentiation" (stating that product specifications will be very similar at equilibrium) hold? Intensive research on this question has conclusively determined that the Principle of Minimum Differentiation does not hold for any well-behaved model. ${ }^{1}$ Thus, as long as we confine product differentiation to one dimension, there will be significant differences in the equilibrium product specifications. However, most goods are defined by a long vector of product attributes, and, a priori, the failure of the Principle of Minimum Differentiation is not clear in multi-attribute competition.

The Principle of Minimum Differentiation fails in one-dimensional models because product similarity increases competition and reduces prices and profits. In multi-attribute models, different possibilities emerge: products can be significantly differentiated along all dimensions ( $\max$-max-. . -max differentiation) or products may have quite different degrees of product differentiation in different dimensions (for example, in three dimensions, maximum differentiation in one dimension and minimum differentiation on the rest, or max-min-min). The logic of the results of the one-dimensional model is not sufficient to show which of these configurations will be the equilibrium in multi-attribute settings.

In this paper we determine the equilibrium configuration in a standard two-dimensional model as max-min. That is, we establish that firms will try to maximally differentiate in one dimension and minimally differentiate in another. We call this the Principle of Maximum-Minimum Differentiation. We further show that when products can be differentiated in three dimensions, firms differentiate maximally in one dimension and minimally in the remaining two. We call this the Principle of Max-Min-Min Differentiation.

In our setup, the disutility of the distance function has different weights in each dimension. These weights measure the importance that consumers place on each attribute of the product. We find that the nature and number of equilibria depend crucially on these weights. For example, in the two-attribute model, when consumers care a lot about the attribute of the first dimension (and therefore place a high weight on it), the max-min equilibrium results, where firms maximally differentiate in the first dimension only. Similarly, when the consumers place a high weight on the second attribute, the min-max equilibrium results, where firms maximally differentiate in the second dimension only. When the weights are roughly comparable, both equilibria exist.

\footnotetext{
${ }^{1}$ See Neven (1985) for a discussion of the necessary conditions for minimal differentiation. Also note that the failure of minimal differentiation does not necessarily imply maximal differentiation. D'Aspremont, Jaskold-Gabszewicz and Thisse (1979) establish a maximal differentiation equilibrium in a one-dimensional variant of Hotelling (1929) by assuming a quadratic disutility of distance (transportation cost) function. Economides (1986b) establishes intermediate (neither minimum nor maximal) differentiation equilibria for a disutility of distance (transportation cost) function of the form $d^{a}, 5 / 3<\mathrm{a}<1.26$. Economides (1984) establishes intermediate differentiation equilibria by allowing for a finite maximal utility (reservation price) for a differentiated good in the original linear disutility of distance function of Hotelling (1929).
}

(c) Blackwell Publishers 1998. 
The same pattern holds in the three-attribute model. The max-min-min equilibrium, where firms maximally differentiate in the first dimension only, occurs when the weight on the first attribute is large. When, in addition, the weight on the second attribute is significant, the min-max-min equilibrium also occurs. When all weights are comparable, the min-min-max equilibrium occurs in addition to the previous two.

The qualitative relationship between weights and type and number of equilibria is very important because it can be used to show a seamless transition from Hotelling's one-characteristic paradigm to models of two and three characteristics. The original one-dimensional model of Hotelling can be embedded in a two-dimensional model where the weight placed by the consumers on the second attribute is negligible. We show that, if this second weight is small, the equilibrium of the two-dimensional model will have maximal differentiation in the first dimension, and no differentiation in the second dimension (max-min). Adding a third attribute that the consumers do not consider important preserves the equilibrium pattern, which now becomes max-min-min. Only when the second weight is significant does a second equilibrium (min-max-min) appear.

The qualitatively different degrees of product differentiation in different dimensions at the equilibria of the two- and three-dimensional models raise the possibility that wrong conclusions will be drawn from empirical observation that does not fully cover all dimensions. For example, in three dimensions, if the maximal differentiation in one dimension remains unobserved, the equilibrium may seem to be one of uniformly minimal differentiation. However, if the only dimension observed is the one that consumers care the most about, then maximal differentiation will be observed. This raises serious concerns about the validity of empirical observation of degrees of product differentiation, since empirical observation is typically incomplete.

All our results are established in the framework of a two-stage game; in the first stage, firms simultaneously choose locations, whereas in the second stage they simultaneously choose prices. Thus, the equilibria we describe are subgameperfect, and firms anticipate the effects of changes in their locations on the equilibrium prices. Intuitively, this game structure captures the fact that prices are more flexible (easier to change) in the short run, whereas product specifications are not; pricing decisions often are made when product specifications cannot be changed (see Salop, 1979; Economides, 1989; and Rao and Steckel, 1995).

In the existing literature, only a few papers have allowed determination of product specifications in two characteristics, notably Economides (1993), Neven and Thisse (1990) and Vandenbosch and Weinberg (1995). ${ }^{2}$ Neven and Thisse

${ }^{2}$ This is in contrast with analysis on the interaction of price and location competition in multidimensional settings without explicit locational determination as in Economides (1986a), or locational determination in Ben Akiva et al. (1989), or two-dimensional models that can be reduced to one-dimensional competition as Lane (1981), Hauser and Shugan (1983), Hauser (1988), and Ansari, Economides and Ghosh (1994).

(C) Blackwell Publishers 1998. 
(1990) investigated product quality and variety decisions of two firms in a two-dimensional product space. They combined the "horizontal" differentiation (ideal point) and "vertical" differentiation (vector-attribute) paradigms, and investigated subgame-perfect equilibria for product and price decisions in a duopoly. Vandenbosch and Weinberg (1994) analyzed a model of two-dimensional vertical (quality) differentiation.

After a working paper version of this article had been circulating, we discovered that Tabuchi (1994) had independently derived similar results for a model of two-dimensional variety differentiation. The primary differences between Tabuchi's model and ours are that we use weights on the disutilities of distance in each attribute and consumers' ideal points are distributed on a square; in contrast, Tabuchi has no weights but allowed for a rectangularly shaped fixed area. We show that there is a formal equivalence between the two models, that is, a rectangle tall in the first dimension in Tabuchi is equivalent to a high preference weight in the first dimension in our model. Nevertheless, the intuitive interpretation of the results is quite different. Further, we provide results for the three-dimensional model.

The remainder of this paper is organized as follows. In Section 2, we present the market environment. In Section 3, we analyze the two dimensional market and derive the price and position equilibria. We extend the model to three dimensions in Section 4. Finally, in Section 5, we conclude with a discussion of our results and provide directions for future research.

\section{THE MODEL}

We describe the model in general terms that are relevant for markets of either two or three attributes. We assume that there are two firms, labelled 1 and 2 , each offering a single $n$-attribute product. The position of a product $i$ can be represented in $n$-dimensional attribute space by an $n$-tuple, $\boldsymbol{\theta}_{i} \in[0,1]^{\mathrm{n}}$. The elements of $\boldsymbol{\theta}_{i}$ provide the position of the product on each of the $n$ attributes. Each consumer is represented by an ideal point that gives the coordinates of the product that the consumer would prefer the most if all products were sold at the same price. A consumer $j$ can therefore be represented by the vector of coordinates of his other ideal point, $\mathbf{A}_{j} \in[0,1]^{\mathrm{n}}$.

Each consumer's utility is a decreasing function of the square of the weighted Euclidean distance between the product specifications and the consumer's ideal point. ${ }^{3}$ Formally, a consumer of type $\mathbf{A}_{j}$ derives the following utility from buying one unit of product $i$ at price $p_{i}$

$$
U\left(\mathbf{A}_{j} ; \boldsymbol{\theta}_{i} ; \mathbf{p}_{i}\right)=Y-\mathbf{w}\left\|\boldsymbol{\theta}_{i}-\mathbf{A}_{j}\right\|^{2}-\mathbf{p}_{i}
$$

\footnotetext{
${ }^{3}$ Models of product differentiation that use a quadratic utility-loss function include D'Aspremont, Jaskold-Gabszewicz, and Thisse (1979), Neven (1985), and Economides (1989). Ideal-point models in marketing assume that preferences are negatively related to the square of the Euclidean distance between the product and the consumer's ideal point (see, e.g., Green and Srinivasan, 1978).
}

(c) Blackwell Publishers 1998 . 
where $Y$ is a positive constant, the same for all consumers and assumed to be high enough so that all consumers buy a differentiated product, and $\mathbf{w}$ is a vector of weights that the consumers attach to attributes. We assume that the $\mathbf{w}$ vector is same across all individuals.

Consumers' ideal points are distributed uniformly over the attribute space; consumers also possess perfect information about brand positions and prices in the market. Firms maximize profits and have zero marginal costs of production. ${ }^{4}$ They compete by following a two-stage process. In the first stage, they simultaneously choose product positions. Once these are determined, they simultaneously choose prices in the second stage. We seek subgame-perfect equilibria of the game implied by this framework. Thus, firms anticipate the impact of location decisions on equilibrium prices. Given this basic model structure, we analyze next the two-dimensional market in detail.

\section{THE TWO-DIMENSIONAL MODEL}

\section{Demand Formulation}

In two dimensions, the joint space of consumers' ideal points and products locations is a unit square. A product $i$ is represented by the vector $\mathbf{V}_{i}=\left(x_{i}, y_{i}\right)$, whereas an arbitrary consumer can be identified by the address $(a, b)$. Without loss of generality, we assume that $y_{2} \geq y_{1}$ and $x_{2} \geq x_{1}$. A consumer's utility for product $i$ takes the form

$$
U_{i}\left(a, b ; x_{i}, y_{i}, p_{i}\right)=Y-w_{1}\left(a-x_{i}\right)^{2}-w_{2}\left(b-y_{i}\right)^{2}-p_{i}, \text { for } i=1,2
$$

The demand for product $i$ is generated by consumers who obtain greater utility from it than from the other product. The locus of consumers who are indifferent between brands 1 and 2 satisfies $U_{1}\left(a, b ; x_{1}, y_{1}, p_{1}\right)=U_{2}\left(a, b ; x_{2}, y_{2}, p_{2}\right)$, which is equivalent to

$$
b(a)=\left[\left(p_{2}-p_{1}\right)+S-2 a w_{1} X\right] /\left[2 w_{2} Y\right]
$$

where $S=w_{1}\left(x_{2}^{2}-x_{1}^{2}\right)+w_{2}\left(y_{2}^{2}-y_{1}^{2}\right), X=x_{2}-x_{1}$, and $Y=y_{2}-y_{1}$. This represents a straight line that partitions the total market (the unit square) into two demand areas for the firms. Given our assumptions regarding the product positions, the area below the separating line represents firm 1's demand and the area above it represents firm 2's demand. The slope of the separating line (b-line) is independent of the prices, but the intercept is not. The location of the line within the unit square depends upon the price difference, $p_{1}-p_{2}$, between the two firms. When firm 1 increases its price (or firm 2 decreases its price), the separating line shifts down reducing the market area for firm 1 . Figure 1 shows the cases

\footnotetext{
${ }^{4}$ Positive constant marginal costs lead to formally equivalent results. The first-order conditions with positive marginal costs are formally equivalent to the first order conditions with zero costs if we redefine prices to be price-cost differences.

(C) Blackwell Publishers 1998.
} 


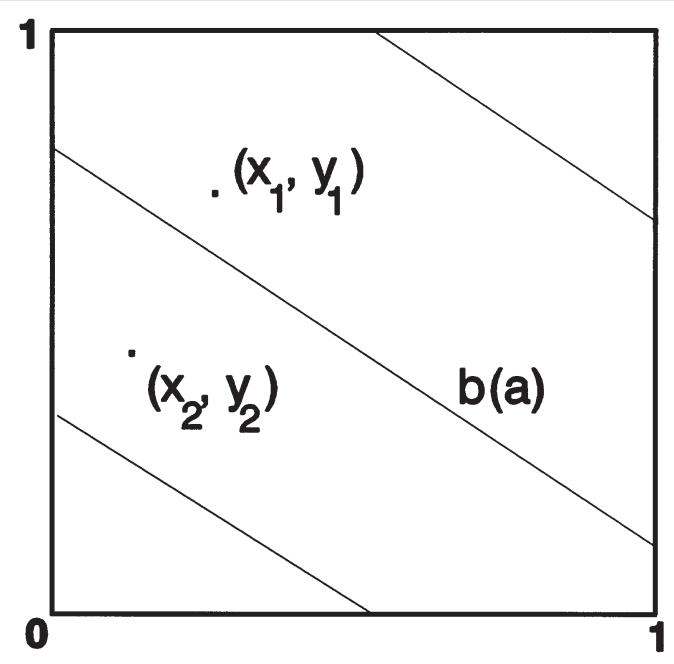

FIGURE 1: Market Areas for the Two Firms.

of Scenario A that arise when $|\partial b / \partial a|<1 \Leftrightarrow w_{1} X<w_{2} Y$, that is, the weighted difference in positions along attribute 2 is greater than the weighted difference in positions along attribute 1. Similarly, Scenario B arises when $w_{2} Y<w_{1} X$.

The demand for firm $1, D_{1}$, is obtained by integrating the $b(a)$ line over the appropriate range of $a$. Because consumers always buy one product or the other, $D_{2}=1-D_{1}$. Assuming zero costs, profits are $\Pi_{1}\left(p_{1}, p_{2} ; x_{1}, y_{1}, x_{2}, y_{2}\right)=p_{1} D_{1}, \Pi_{2}\left(p_{1}\right.$, $\left.p_{2} ; x_{1}, y_{1}, x_{2}, y_{2}\right)=p_{2} D_{2}$.

\section{Scenario A}

We first analyze Scenario A and show how the demand expressions and profit functions depend upon the relative price difference between the firms. We fix the positions of both brands and the price of firm $2, p_{2}$. As price $p_{1}$ decreases, the $b$ line that separates the market areas shifts upward. The three lines in Figure 1 represent cases $1 \mathrm{~A}, 2 \mathrm{~A}$, and $3 \mathrm{~A}$ respectively. The demand expressions for each case are summarized below. We label the demand expressions for firm $i$ in case $k$ as $D_{i}^{\mathrm{k}}$.

Case $1 A$. When $0 \leq\left(p_{2}-p_{1}+S\right) \leq 2 w_{1} X$, the demand of firm 1 is $D_{1}{ }^{1 \mathrm{~A}} \equiv\left(p_{2}-p_{1}+S\right)^{2} /\left(8 w_{1} w_{2} X Y\right)$.

Case $2 A$. When $2 w_{1} X \leq\left(p_{2}-p_{1}+S\right) \leq 2 w_{2} Y$, the demand of firm 1 is $D_{1}{ }^{2 \mathrm{~A}} \equiv\left(p_{2}-p_{1}+S-w_{1} X\right) /\left(2 w_{2} Y\right)$.

Case $3 A$. When $2 w_{2} Y \leq\left(p_{2}-p_{1}+S\right) \leq 2\left(w_{1} X+w_{2} Y\right)$, the demand of firm 1 is $D_{1}^{3 \mathrm{~A}} \equiv\left(p_{2}-p_{1}+S-w_{1} X-w_{2} Y\right)^{2} /\left(8 w_{1} w_{2} X Y\right)$. 


\section{Scenario B}

In Scenario B, the product positions satisfy $w_{1} Y<w_{2} X$; we again have three cases for the demand. These demand expressions and price domains are summarized below.

Case $1 B$. When $0 \leq\left(p_{2}-p_{1}+S\right) \leq 2 w_{2} Y$, the demand of firm 1 is $D_{1}^{1 B}=D_{1}^{1 A}$.

Case $2 B$. When $2 w_{2} Y \leq p_{2}-p_{1}+S \leq 2 w_{1} X$, the demand of firm 1 is $D_{1}{ }^{2 B} \equiv\left(p_{2}-p_{1}+S-w_{2} Y\right) /\left(2 w_{1} X\right)$.

Case $3 B$. When $2 w_{1} X \leq\left(p_{2}-p_{1}+S\right) \leq 2\left(w_{1} X+w_{2} Y\right)$, the demand of firm 1 is $D_{1}^{3 \mathrm{~B}}=D_{1}{ }^{3 \mathrm{~A}}$.

It is easy to check that the demand expressions are continuous across the different price domains. The different segments of $D_{2}$ can be derived in a manner analogous to that for $D_{1}$.

\section{Price Equilibrium}

In this section, we show that a unique, noncooperative price equilibrium exists for any pair of product positions (chosen by the two firms in the first stage) in both two and three dimensions, and we calculate the equilibrium prices. The main step in proving existence is in establishing that each firm's profit function is quasi-concave in its own price. The concavity properties of the profit function depend on the choice of the utility function and the distribution of consumer preferences. Caplin and Nalebuff (1991) have established twin restrictions on utility functions and preference distributions that guarantee existence of price equilibria for a number of firms with $n$-dimensional product specifications. Our utility function (2) is a special case of the general utility function of Caplin and Nalebuff (Assumption A1, p. 29). In addition, the uniform distribution of consumer preferences is concave and conforms with the $\rho$-concavity conditions employed in Caplin and Nalebuff. Hence our model satisfies Assumptions A1 and A2, of Caplin and Nalebuff. Then, from their Theorems 1 and 2, a price equilibrium exists in our model for any pair of positions. Because the profit function is twice differentiable and the distribution of preferences is concave (and therefore log-concave), Caplin and Nalebuff's uniqueness result (Proposition 6, p. 42) ensures that the price equilibrium is unique for each pair of product positions. What remains is to calculate the equilibrium prices for each pair of locations. This has to be done for each case in each scenario. The equilibrium price functions are obtained by solving the first-order conditions of the profit functions, $\partial \Pi_{1} / \partial p_{1}=\partial \Pi_{2} / \partial p_{2}=0$, and checking the second-order conditions to ensure a positive price solution. ${ }^{5}$ We describe these equilibria in the appendix

\footnotetext{
${ }^{5}$ Because of the complexity of the problem, we solved this system of equations numerically. Although we were unable to obtain closed form solutions, we implemented our numerical routine with a wide variety of starting values so that we have maximum confidence in our results.

(C) Blackwell Publishers 1998.
} 
of an unabridged working paper version of this article available upon request from the authors.

We now illustrate the relationship between our two-dimensional model and Tabuchi's (1994) formulation. ${ }^{6}$ Tabuchi models location-price equilibria in a rectangular product space, but assumes that both attributes are equally important for consumers. We assume that the two-dimensional product space is a unit square, but associate different weights along the two dimensions. We find that the demand and profit expressions as well as the price equilibria are equivalent in these two formulations via a set of transformations on the product coordinates and attribute weights. Specifically, let $\left(x_{1}, y_{1}, x_{2}, y_{2}\right)$ and $\left(w_{1}, w_{2}\right)$ be the product coordinates and attribute weights, respectively, in our formulation, and let $\left(X_{1}\right.$, $Y_{1}, X_{2}, Y_{2}$ ) be the product coordinates in Tabuchi's model, where consumers are distributed uniformly in the rectangular product space $[0, c] \times[0,1 / c]$. The transformation that establishes equivalence is: $x_{1}=X_{1} / c, x_{2}=X_{2} / c, y_{1}=c Y_{1}$ and $y_{2}=c Y_{2} ; w_{1}=c^{2}$, and $w_{2}=1 / c^{2}$. Thus, a thinner and taller rectangle in Tabuchi is formally equivalent to our unit square with more weight given to the second (i.e., the vertical) characteristic. Because the demand and profit expressions are the same, so are the equilibrium prices for equivalent positions in the two models.

\section{Product Equilibria}

We now establish the subgame-perfect equilibrium positions of firms. With subgame perfection, firms anticipate the equilibrium prices in the subgames. We can write profits in the locations stage as

$$
\begin{gathered}
\prod_{i}^{\ell}\left(x_{1}, y_{1}, x_{2}, y_{2}\right) \equiv \Pi_{i}\left[p_{1}{ }^{*}\left(x_{1}, y_{1}, x_{2}, y_{2}\right), p_{2}{ }^{*}\left(x_{1}, y_{1}, x_{2}, y_{2}\right)\right. \\
\left.x_{1}, y_{1}, x_{2}, y_{2}\right], i=1,2
\end{gathered}
$$

Thus, a change in location has two effects on profits: a direct effect and an indirect effect through prices. ${ }^{7}$

Depending on the ratio of the weights $w \equiv w_{2} / w_{1}$, there are either one or two location equilibria (and their mirror images). At all equilibria there is minimum differentiation in one dimension and maximum differentiation in the other. The first candidate equilibrium is $\left(x_{1}{ }^{*}, y_{1}{ }^{*}\right)=(1 / 2,0),\left(x_{2}{ }^{*}, y_{2}{ }^{*}\right)=(1 / 2,1)$, that is, firms are located in the middle of the horizontal segments of the box, implying minimum differentiation in $\mathrm{x}$ and maximum differentiation in $\mathrm{y}$. We call this the min-max equilibrium. The second candidate equilibrium is $\left(x_{1}{ }^{* *}, y_{1}{ }^{* * *}\right)=(0,1 / 2)$, $\left(x_{2}{ }^{* *}, y_{2}{ }^{* * *}\right)=(1,1 / 2)$, that is, firms are located at the middle points of the vertical

\footnotetext{
${ }^{6}$ We are grateful to an anonymous referee for pointing out the equivalence.

${ }^{7}$ Essentially the indirect effect is through the price of the opponent: $d \Pi_{i}^{\ell}-/ d x_{i}=\partial \Pi_{i} / \partial x_{i}+$ $\left(\partial \Pi_{i} / \partial p_{i}\right)\left(d p_{i}^{*} / d x_{i}\right)+\left(\partial \Pi_{i} / \partial p_{j}\right)\left(d p_{j}^{*} / d x_{i}\right)=\partial \Pi_{i} / \partial x_{i}+\left(\partial \Pi_{i} / \partial p_{j}\right)\left(d p_{j}^{*} / d x_{i}\right)$, since $\partial \Pi_{i} / \partial p_{i}=0$ at the Nash equilibrium of the price subgame.

(c) Blackwell Publishers 1998 .
} 
segments of the box, implying minimum differentiation in $y$ and maximum differentiation in $x$. We call this the max-min equilibrium.

We find that for $w<0.406$ only the max-min equilibrium $\left[\left(x_{1}^{* * *}, y_{1}^{* * *}\right)=(0,1 / 2)\right.$, $\left.\left(x_{2}^{* *}, y_{2}^{* * *}\right)=(1,1 / 2)\right]$ exists; for $w>1 / 0.406=2.46$, only the min-max equilibrium $\left[\left(x_{1}{ }^{*}, y_{1}{ }^{*}\right)=(1 / 2,0),\left(x_{2}{ }^{*}, y_{2}{ }^{*}\right)=(1 / 2,1)\right]$ exists; and for $0.406<w<2.46$, both the max-min and the min-max equilibria exist. ${ }^{8}$ The nature of the best responses underlying these equilibria are shown in Figure 2. The arrows in these gradient plots show the direction in which the profit function of firm 1 increases, when

(a) $w=2.5$

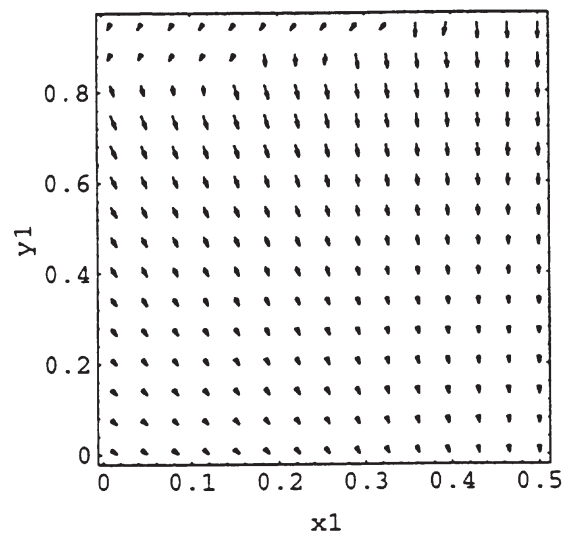

(c) $w=0.7$

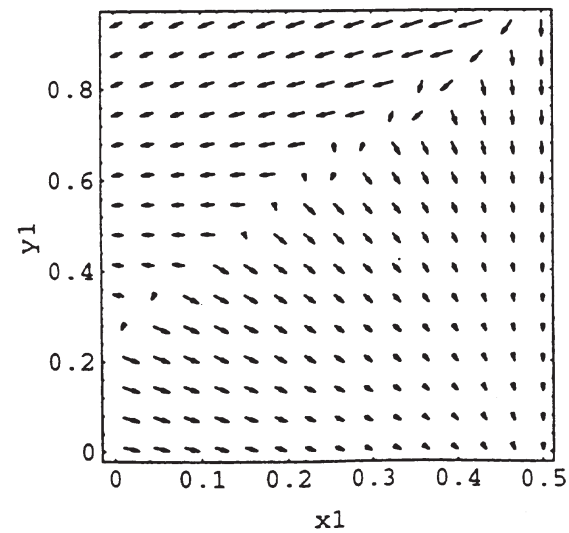

(b) $\quad w=1$

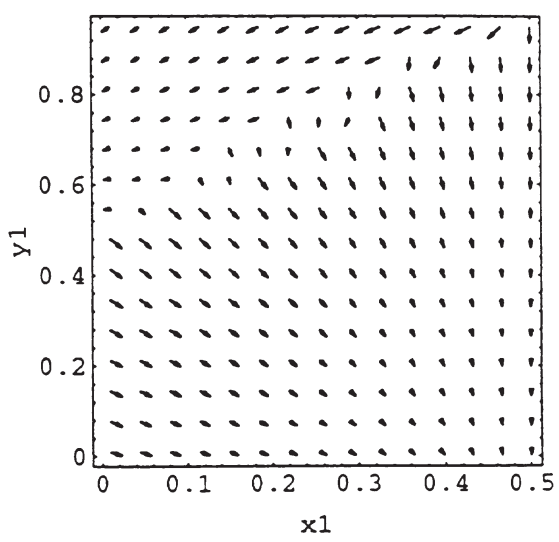

(d) $w=0.4$

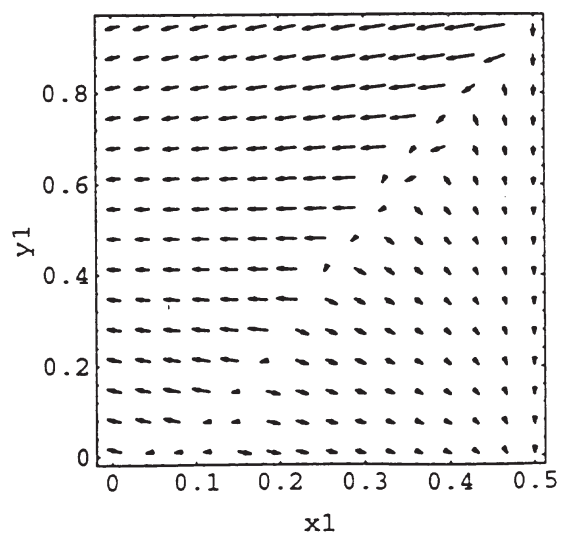

FIGURE 2: Gradient of Profit Function for Firm 1. Firm 2 is Located at $(1 / 2,1)$.

${ }^{8}$ The method of our proof is detailed in the product-equilibrium section later in the paper. 
firm 2 is located at $(1 / 2,1)$. Remembering the definition of $w, w=w_{1} / w_{2}$, note that the min-max equilibrium exists when $w_{2}$ is relatively large, and similarly, the max-min equilibrium exists when $\mathrm{w}_{1}$ is relatively large. When $w_{1}$ and $w_{2}$ are roughly of similar magnitude, as in Figure 2(c), both equilibria exist. When one weight is much larger than the other, there is only one equilibrium where maximal differentiation occurs in the dimension that corresponds to the higher weight.

At both equilibria, the firms share the market equally. At the first equilibrium, prices for both brands are $p_{1}=p_{2}=w_{2}$ and profits are $\Pi_{1}=\Pi_{2}=w_{2} / 2$; at the second equilibrium, prices are $p_{1}=p_{2}=w_{1}$ and profits are $\Pi_{1}=\Pi_{2}=w_{1} / 2$. Therefore, when the weights differ, and when both equilibria exist, both firms are better-off at the equilibrium that corresponds to maximal differentiation in the product dimension for which the consumers care most. Firms are likely to gravitate to that equilibrium.

Positions implying maximal differentiation on both attributes (max-max) are not equilibrium positions, even though both firms have profits equal to the equilibrium profits when they are maximally differentiated on both attributes. Given that its opponent has located at the corner of the square, a firm has a unilateral incentive to deviate from the diametrically opposite corner and move inwards thereby increasing its market share. Such an inward move in one attribute is based on incentives that are analogous to those in the one-dimensional Hotelling (1929) model with linear transportation costs. In that model, profits are equal for all symmetric locations, but each firm has a unilateral incentive to move toward the other firm. The two opposing forces, one driving firms apart due to price competition, and the other bringing them together due to market-share dynamics, are resolved in favor of market-share forces. In contrast, in the one-dimensional model with quadratic costs, where again the opposing forces operate on a single attribute, the resolution is in favor of price competition, and the equilibrium is at maximal differentiation. When there are two or more dimensions, the two opposing forces resolve along different dimensions. Due to the dominance of price competition, we see maximal differentiation along the most important attribute, whereas the market-share effect encourages firms to occupy central positions on the less important attribute.

An example of a product category that exhibits max-min differentiation is ice cream. Consider a single flavor, such as chocolate. Most of the important differences among brands are dictated by their fat and sugar contents. These characteristics govern both flavor and how healthy (or, perhaps more accurately, unhealthy) each brand is. A trip to the grocery store with a casual inspection of the nutrition labels will reveal that the amount of fat per 100-gram serving (approximately $1 / 2$ to $3 / 4$ cup, depending on the ice cream's density) varies from none for the fat-free brands to about 22 grams for the super premium brands (e.g., Haagen Daas). Yet all brands tend to have in the neighborhood of 25 grams of carbohydrates or sugar. 
It is natural to ask whether this pattern of equilibria generalizes to higher dimensions. In higher dimensions, will competitors differentiate on more than one attribute or continue to differentiate only on one attribute? Will we continue to get just two equilibria or will the number of equilibria depend on the dimensionality of the product space? To answer these questions, we analyze a three-dimensional market in the next section.

\section{THE THREE DIMENSIONAL MODEL}

\section{Demand Formulation}

In three dimensions, the joint space of consumers and product locations is a unit cube. A product $i$ is represented by the vector $\boldsymbol{\theta}_{i}=\left(x_{i}, y_{i}, z_{i}\right)$ whereas an ideal point for consumer $j$ is denoted by $\mathbf{A}_{j}=(a, b, c)$. We continue to assume that $x_{2} \geq x_{1}, y_{2} \geq y_{1}, z_{2} \geq z_{1}$, and that the attribute weights, $w_{1}, w_{2}, w_{3}$, are constant across consumers. Thus, the utility of consumer $\mathbf{A}_{j}$ when he buys one unit of product $\boldsymbol{\theta}_{i}$ is

$$
U_{i}\left(a, b, c ; x_{i}, y_{i}, z_{i}\right)=Y-w_{1}\left(a-x_{i}\right)^{2}-w_{2}\left(b-y_{i}\right)^{2}-w_{3}\left(c-z_{i}\right)^{2}-\mathrm{p}_{\mathrm{i}} \quad i=1,2
$$

The market areas are given by three-dimensional regions of the cube separated by a plane, rather than two-dimensional regions of a square separated by a line as in the two-dimensional case we discussed earlier. The locus of consumers on this plane, who are indifferent between buying from either firm, is given by

$$
c(a, b)=\left(p_{2}-p_{1}+S-2 a w_{1} X-2 b w_{2} Y\right) /\left(2 w_{3} Z\right)
$$

where $S=w_{1}\left(x_{2}^{2}-x_{1}^{2}\right)+w_{2}\left(y_{2}^{2}-y_{1}^{2}\right)+w_{3}\left(z_{2}^{2}-z_{1}^{2}\right), X=x_{2}-x_{1}, Y=y_{2}-y_{1}, Z=z_{2}-z_{1}$, and $(a, b) \in[0,1] \times[0,1]$ are the coordinates of the consumer in the first two dimensions. The region below (above) the plane is composed of customers of product 1 (2). As before, when firm 1 decreases its price $p_{1}$ (or firm 2 increases $\mathrm{p}_{2}$ ), the plane shifts upwards, thereby increasing firm 1's demand, $\mathrm{D}_{1}$. The demand for firm 1 is obtained by integrating the $c(a, b)$ plane over the appropriate range of $a$ and $b$. Since consumers buy one product or the other, $D_{2}=1-D_{1}$. As the demand expressions for the two firms depend upon the position and orientation of the separating plane, the demand expressions change whenever the indifference plane shifts its location and passes through a corner of the unit cube. To capture the dependence of the demand expressions on the relationship between prices and firm locations, we first distinguish between twelve scenarios that are characterized by the locations of the firms.

The defining features of these scenarios are:

Scenario $1 A . w_{1} X \leq w_{2} Y \leq\left(w_{1} X+w_{2} Y\right) \leq w_{3} Z$;

Scenario $1 B . w_{1} X \leq w_{2} Y \leq w_{3} Z \leq\left(w_{1} X+w_{2} Y\right)$;

Scenario $2 A . w_{1} X \leq w_{3} Z \leq\left(w_{1} X+w_{3} Z\right) \leq w_{2} Y$; 
Scenario $2 B . w_{1} X \leq w_{3} Z \leq w_{2} Y \leq\left(w_{1} X+w_{3} Z\right)$;

Scenario $3 A . w_{2} Y \leq w_{1} X \leq\left(w_{1} X+w_{2} Y\right) \leq w_{3} Z$;

Scenario $3 B . w_{2} Y \leq w_{1} X \leq w_{3} Z \leq\left(w_{1} X+w_{2} Y\right)$;

Scenario $4 A . w_{2} Y \leq w_{3} Z \leq\left(w_{2} Y+w_{3} Z\right) \leq w_{1} X$;

Scenario $4 B$. $w_{2} Y \leq w_{3} Z \leq w_{1} X \leq\left(w_{2} Y+w_{3} Z\right)$;

Scenario $5 A . w_{3} Z \leq w_{1} X \leq\left(w_{1} X+w_{3} Z\right) \leq w_{2} Y$;

Scenario $5 B . w_{3} Z \leq w_{1} X \leq w_{2} Y \leq\left(w_{1} X+w_{3} Z\right)$;

Scenario $6 A . w_{3} Z \leq w_{2} Y \leq\left(w_{2} Y+w_{3} Z\right) \leq w_{1} X$; and

Scenario $6 B . w_{3} Z \leq w_{2} Y \leq w_{1} X \leq\left(w_{2} Y+w_{3} Z\right)$.

We now focus on the dependence of the demand expressions and profit functions on the relative price difference between the firms. Within each scenario (configuration of attribute weights and product positions), we identify seven cases that are distinguished by the difference in the prices, $p_{2}-p_{1}$. We now present the demand expressions under Scenario 1A, and discuss the other scenarios in the Appendix.

In Scenario $1 \mathrm{~A}$, for any fixed price $p_{2}$, if $p_{1}$ is such that $p_{2}-p_{1}+S \leq 0$, then Firm 1 has nonpositive demand. In order to calculate the demand segment $D_{1}{ }^{1 \mathrm{~A}}$, we need to define the region of integration. The separating plane intersects the $(a, b)$ plane in a straight line given by blimit $=\left(p_{2}-p_{1}+S-2 a w_{1} X\right) /\left(2 w_{2} Y\right)$. This straight line, blimit, intersects the $a$ axis at point aint $=\left(p_{2}-p_{1}+S\right) /\left(2 w_{1} X\right)$. Now, when $p_{1}$ is reduced so that $0 \leq L \leq 2 X$, where $L=\left(p_{2}-p_{1}+S\right)$, the separating plane intersects all three axes as in Case 1, Figure 3.

Case $1 A$. When $0 \leq \mathrm{L} \leq 2 w_{1} X$, demand for firm 1 is

$$
D_{1}^{1 A}=\int_{0}^{\text {aint }} \int_{0}^{\text {blimit }} c(a, b) d b d a=L^{3} / H
$$

where $H=48 w_{1} w_{2} w_{3} X Y Z$

When $p_{1}$ is further decreased, the separating plane passes through the corner $(1,0,0)$ of the product space and as shown in Case 2, Figure 3.

Case $2 A$. When $2 w_{1} X \leq L \leq 2 w_{2} Y$, demand is

$$
D_{1}^{2 A}=\int_{0}^{1} \int_{0}^{b l i m i t} c(a, b) d b d a=D_{1}^{1 A}-\left(L-w_{1} X\right)^{3} / H
$$

When $p_{1}$ is further reduced, the plane moves up and crosses the corner $(0,1,0)$. 
Case $3 A$. When $2 w_{2} Y \leq L \leq 2\left(w_{1} X+w_{2} Y\right)$

$$
\begin{gathered}
D_{1}^{3 A}=\int_{0}^{\text {aint }} \int_{0}^{1} c(b, a) d b d a+ \\
\int_{\text {aint } 1}^{1} \int_{0}^{b l i m i t} c(b, a) d b d a=D_{1}^{2 A}-\left(L-2 w_{2} Y\right)^{3} / H
\end{gathered}
$$

where aint $1=\left(L-2 w_{2} Y\right) /\left(2 w_{1} X\right)$ is the intercept of the blimit line with the line $b=1$.

\section{Scenario 1}
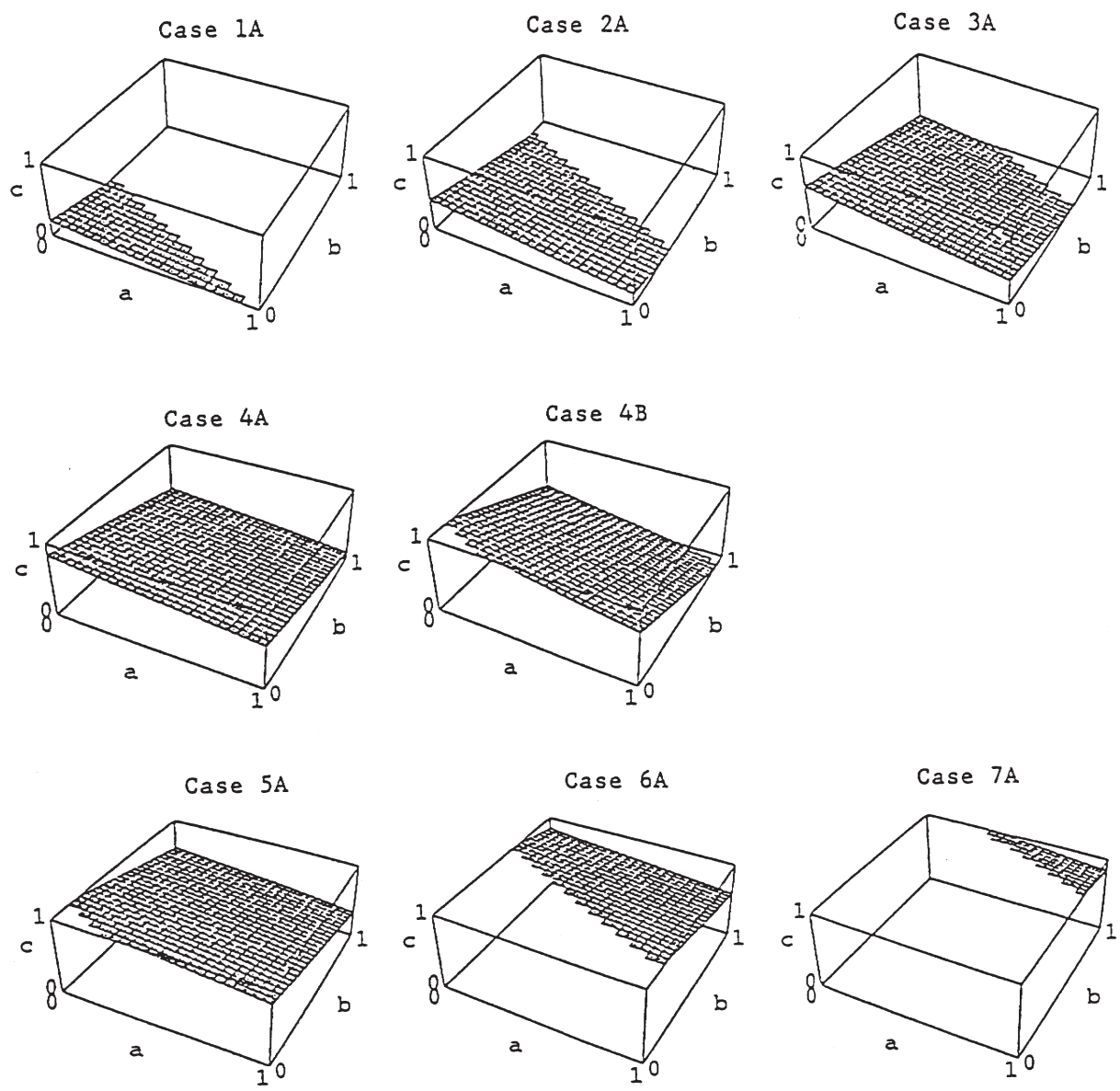

FIGURE 3: Market Areas (Volumes) in Three Dimensions. 
With further reduction in $p_{1}$, we have Case $4 \mathrm{a}$, Figure 3 , where the indifference plane intersects the vertical faces of the unit cube:

Case $4 A$. When $2\left(w_{1} X+w_{2} Y\right) \leq L \leq 2 w_{3} Z$,

$$
D_{1}^{4 A}=\int_{0}^{1} \int_{0}^{1} c(b, a) d b d a=D_{1}^{3 A}+\left[L-2\left(w_{1} X+w_{2} Y\right)\right]^{3} / H,
$$

which simplifies further to a linear function in $p_{1}$ given by

$$
D_{1}^{4 A}=\left(L-w_{1} X-w_{2} Y\right) /\left(2 w_{3} Z\right) .
$$

When $p_{1}$ is further reduced so that the separating plane moves past the $(0,0,1)$ corner, we have:

Case $5 A$. When $2 w_{3} Z \leq L \leq 2\left(w_{1} X+w_{3} Z\right)$

$$
\begin{aligned}
& D_{1}^{5 A}=\int_{0}^{\text {aint } 2} \int_{0}^{\text {blimit } 1} d b d a+ \\
& \int_{0}^{\text {aint2 }} \int_{\text {blimit } 1}^{1} c(b, a) d b d a+\int_{\text {aint } 2}^{1} \int_{0}^{1} c(b, a) d b d a
\end{aligned}
$$

where blimit $1=\left(L-2 a w_{1} X-2 w_{3} Z\right) /\left(2 w_{2} Y\right)$ is the line of intersection of $c(a, b)$ with the plane $c=1$. The point aint2 is obtained by substituting $b=0$ in blimit 1 . Hence, aint $2=\left(L-2 w_{3} Z\right) /\left(2 w_{1} X\right)$, whereas bint $2=\left(L-2 w_{3} Z\right) /\left(2 w_{2} Y\right)$ is obtained by setting $a=0$ in blimit 1 . The demand expression then is

$$
D_{1}^{5 A}=D_{1}^{4 A}-\left(L-2 w_{3} Z\right)^{3} / H
$$

Next, on further reduction in $p_{1}$, the plane passes past $(1,0,1)$.

Case 6A. When $p_{1}$ satisfies $2\left(w_{1} X+w_{3} Z\right) \leq L \leq 2\left(w_{2} Y+w_{3} Z\right)$, we have

$$
D_{1}^{6 A}=\int_{0}^{1} \int_{0}^{b l i m i t 1} d b d a+\int_{0}^{1} \int_{b l i m i t 1}^{1} c(b, a) d b d a
$$

which reduces to

$$
D_{1}^{6 \mathrm{~A}}=D_{1}{ }^{5 \mathrm{~A}}+\left[L-2\left(w_{1} X+w_{2} Y\right)\right]^{3 / H}
$$

Finally, we calculate the point of intersection of blimit 1 with the line $c=1$ and $b=1$ to get aint $3=\left[L-2\left(w_{2} Y+w_{3} Z\right)\right] /\left(2 w_{1} X\right)$. Now, as shown in Case 7 , Figure 3, we have:

Case $7 A$. When $2\left(w_{2} Y+w_{3} Z\right) \leq L \leq 2\left(w_{1} X+w_{2} Y+w_{3} Z\right)$, the demand is given by

$$
D_{1}^{7 A}=\int_{0}^{\text {aint } 3} \int_{0}^{1} d b d a+\int_{\text {aint } 3}^{1} \int_{0}^{b l i m i t 1} d b d a+\int_{\text {aint } 3}^{1} \int_{b \operatorname{limit} 1}^{1} c(b, a) d b d a
$$


This reduces to

$$
D_{1}{ }^{7 \mathrm{~A}}=D_{1}^{6 \mathrm{~A}}+\left[L-2\left(w_{2} Y+w_{3} Z\right)\right]^{3 / H}
$$

This completes Scenario 1A. The different segments of $D_{2}$ can be derived in a manner analogous to that for $D_{1}$. It is easy to show that all demand expressions are continuous across the different price domains (cases).

\section{Price Equilibrium}

Following the arguments based on Caplin and Nalebuff (1991) given for the two-dimensional model in Section 3, a unique price equilibrium exists for each set of product positions in three dimensions. We therefore begin by describing the equilibrium price expressions within each case of Scenario $1 \mathrm{~A}$.

Case $1 A$. The demand for firm 1 is given by $D_{1}^{1 \mathrm{~A}}$ and for firm 2 is given by $D_{2}{ }^{1 \mathrm{~A}}=1-D_{1}{ }^{1 \mathrm{~A}}$. The first-order conditions yield three solutions. We eliminate infeasible solutions to obtain the equilibrium prices

$$
p_{1}{ }^{1 A^{*}}=\left(S^{2}+K S+K^{2}\right) /(15 K), p_{2}{ }^{1 \mathrm{~A}^{*}}=\left(4 S^{2}-11 K S+4 K^{2}\right) /(15 K)
$$

where $K=\left\{S^{3}+600 w_{1} w_{2} w_{3} X Y Z+20\left[3 w_{1} w_{2} w_{3} X Y Z\left(S^{3}+300 w_{1} w_{2} w_{3} X Y Z\right)\right]^{1 / 2}\right\}^{1 / 3}$

The above equilibrium prices apply to product positions that satisfy $0 \leq p_{2}{ }^{1 \mathrm{~A}^{*}}$ $-p_{1}{ }^{1 \mathrm{~A}^{*}}+S<2 w_{1} X$. Positions that result in Case $1 \mathrm{~A}$ also satisfy $w_{1} X \leq w_{2} Y \leq\left(w_{1} X\right.$ $\left.+w_{2} Y\right) \leq w_{3} Z$. We define the pairs of locations that satisfy these conditions (and therefore result in Case 1A) as $R_{1 A}$. For fixed values of the weights, $R_{1 A}$ is a subset of the six-dimensional hypercube $[0,1]^{6}$. The condition $p_{2}{ }^{1 A^{*}}-p_{1}{ }^{1 A^{*}}+S \geq 0$ is always true, whereas $p_{2}{ }^{1 A^{*}}-p_{1}{ }^{1 A^{*}}+S<2 w_{1} X$ is satisfied if

$$
10 w_{1}^{2} X^{2}>12 w_{2} w_{3} Y Z+3 w_{1} S X
$$

Case $2 A$. The first-order conditions yield two solutions. After eliminating the one that gives negative prices, the equilibrium prices are

$$
\begin{gathered}
p_{1}{ }^{2 A^{*}}=\left[9\left(S-w_{1} X\right)\left(4 w_{2} w_{3} Y Z-w_{1}^{2} X^{2}\right)+J w_{1}^{2} X^{2}+108 J w_{2} w_{3} Y Z\right] / \\
{\left[24\left(12 w_{2} w_{3} Y Z-w_{1} X^{2}\right)\right]} \\
p_{2}{ }^{2 A^{*}}=\left[9\left(S-w_{1} X\right)\left(-20 w_{2} w_{3} Y Z+w_{1}^{2} X^{2}\right)-J w_{1}^{2} X^{2}+972 J w_{2} w_{3} Y Z\right] / \\
{\left[24\left(12 w_{2} w_{3} Y Z-w_{1} X^{2}\right)\right]}
\end{gathered}
$$

where $J=\left(9 S^{2}-18 w_{1} S X-15 w_{1}^{2} X^{2}+288 w_{2} w_{3} Y Z\right)^{1 / 2}$

Substitution of equilibrium prices in the defining condition of Case $2 \mathrm{~A}$, results in $2 w_{1} X \leq p_{2}{ }^{2 A^{*}}-p_{1}{ }^{2 A^{*}}+S<2 w_{2} Y$. The left-hand side of this inequality is satisfied when condition (3) fails, that is, when $\left(10 w_{1}^{2} X^{2} \leq 12 w_{2} w_{3} Y Z+3 w_{1} S X\right)$, whereas the right-hand side of the inequality is satisfied when

$$
3 S+J<24 w_{2} Y-9 w_{1} X
$$

These two conditions, in conjunction with $w_{1} X \leq w_{2} Y \leq\left(w_{1} X+w_{2} Y\right) \leq w_{3} Z$, define region $R_{2 A}$, of location pairs for which $p_{1}{ }^{2 A^{*}}$ and $p_{2}{ }^{2 A^{*}}$ define an equilibrium. 
Case $3 A$. The equilibrium prices can be obtained by solving the system of equations below.

$$
\begin{gathered}
\left(p_{1}+p_{2}\right) M+1=0 \\
p_{1} M+\left(p_{2}-p_{1}+S-w_{1} X-w_{2} Y\right) /\left(2 w_{3} Z\right)-N^{3} /\left(48 w_{1} w_{2} w_{3} X Y Z\right)=0
\end{gathered}
$$

where $N=\left(p_{2}-p_{1}+S-2 w_{1} X-2 w_{2} Y\right)$ and $M=N^{2} /\left(16 w_{1} w_{2} w_{3} X Y Z\right)-1 /\left(2 w_{3} Z\right)$.

We were unable to obtain closed-form solutions to this set of equations. The equilibrium prices for this case apply to product locations which satisfy $2 w_{2} Y \leq$ $\left(p_{2}{ }^{3 A^{*}}-p_{1}{ }^{3 A^{*}}+S\right)<2\left(w_{1} X+w_{2} Y\right)$. The left-hand side for the above inequality is satisfied when (4) fails and the right-hand side is satisfied when

$$
4 w_{1} X+4 w_{2} Y-2 w_{3} Z>S
$$

These conditions, along with $w_{1} X \leq w_{2} Y \leq\left(w_{1} X+w_{2} Y\right) \leq w_{3} Z$, define the region $R_{3 \mathrm{~A}}$ of product coordinates for which the above equations yield equilibrium prices.

Case 4A. Equilibrium prices are

$$
\begin{aligned}
p_{1}{ }^{4 \mathrm{~A}^{*}}= & \left(S-w_{1} X-w_{2} Y+2 w_{3} Z\right) / 3, p_{2}{ }^{4 A^{*}}= \\
& \left(-S+w_{1} X+w_{2} Y+4 w_{3} Z\right) / 3
\end{aligned}
$$

Substitution of equilibrium prices in the defining condition of case $4 \mathrm{~A}$, results in $2\left(w_{1} X+w_{2} Y\right) \leq p_{2}{ }^{4 \mathrm{~A}^{*}}-p_{1}{ }^{4 \mathrm{~A}^{*}}+S<2 w_{3} Z$. The left-hand side of this inequality is satisfied when condition (5) fails, that is, $4\left(w_{1} X+w_{2} Y\right)-2 w_{3} Z \leq S$, whereas the right-hand side is satisfied when

$$
S<4 w_{3} Z-2\left(w_{1} X+w_{2} Y\right)
$$

These two conditions, along with $w_{1} X \leq w_{2} Y \leq\left(w_{1} X+w_{2} Y\right) \leq w_{3} Z$, define region $R_{4 A}$.

Case 5A. The equilibrium prices can be obtained by solving the system of equations below.

$$
\begin{gathered}
\left(p_{1}+p_{2}\right) B+1=0 \\
p_{1} B+\left(p_{2}-p_{1}+S-w_{1} X-w_{2} Y\right) /\left(2 w_{3} Z\right)-A^{3} /\left(48 w_{1} w_{2} w_{3} X Y Z\right)=0
\end{gathered}
$$

where $A=\left(p_{2}-p_{1}+S-2 w_{3} Z\right)$ and $B=A^{2} /\left(16 w_{1} w_{2} w_{3} X Y Z\right)-1 /\left(2 w_{3} Z\right)$.

We were unable to obtain closed-form solutions for this set of equations. The equilibrium prices for this case apply to product locations which satisfy $2 w_{3} \mathrm{Z} \leq$ $\left(p_{2}{ }^{5 A^{*}}-p_{1}{ }^{5 A^{*}}+S\right)<2\left(w_{1} X+w_{3} Z\right)$. The left-hand side for this inequality is satisfied when (6) fails and the right-hand side is satisfied when

$$
3 S-E>15 w_{1} X+16 w_{3} Z-18 w_{2} Y
$$

These conditions, together with $w_{1} X \leq w_{2} Y \leq\left(w_{1} X+w_{2} Y\right) \leq w_{3} Z$, define the region $R_{5 A}$ of product coordinates for which the above equations yield equilibrium prices. 
Case 6A. The first-order conditions yield two solutions. After eliminating the one with negative prices we have the following equilibrium prices:

$$
\begin{gathered}
p_{1}{ }^{6 A^{*}}=\left[9 I\left(20 w_{2} w_{3} Y Z-w_{1}{ }^{2} X^{2}\right)-E w_{1}{ }^{2} X^{2}+\right. \\
\left.972 E w_{2} w_{3} Y Z\right] /\left[24\left(12 w_{2} w_{3} Y Z-w_{1} X^{2}\right)\right] \\
p_{2}{ }^{6 A^{*}}=\left[9 I\left(-4 w_{2} w_{3} Y Z+w_{1}{ }^{2} X^{2}\right)+E w_{1}{ }^{2} X^{2}+\right. \\
\left.108 \mathrm{E} w_{2} w_{3} Y Z\right] /\left[24\left(12 w_{2} w_{3} Y Z-w_{1} X^{2}\right)\right]
\end{gathered}
$$

where $I=S-w_{1} X-2 w_{2} Y-2 w_{3} Z$ and $E=\left[9 I^{2}+24\left(14 w_{2} w_{3} Y Z-w_{1}^{2} X^{2}\right)\right]^{1 / 2}$.

These equilibrium expressions apply for product positions that satisfy $2\left(w_{1} X+\right.$ $\left.w_{3} Z\right) \leq p_{2}{ }^{6 \mathrm{~A}^{*}}-p_{1}{ }^{6 \mathrm{~A}^{*}}+S<2\left(w_{3} Z+w_{2} Y\right)$. The left-hand side of this inequality is true when (7) fails whereas the right-hand side is true when

$$
3 S-E \leq 6 w_{3} Z+6 w_{2} Y-9 w_{1} X
$$

Case 7A. The first-order conditions yield three solutions. After eliminating the complex roots, the equilibrium prices are given by

$$
\begin{gathered}
p_{1}{ }^{7 \mathrm{~A}^{*}}=\left(128 T^{2}-11 F T+128 F^{2}\right) /(15 F), p_{2}{ }^{7 \mathrm{~A}^{*}}= \\
\left(T^{2}+T F+F^{2}\right) /(15 F)
\end{gathered}
$$

where $T=\left(2 w_{1} X+2 w_{2} Y+2 w_{3} Z-S\right)$ and

$$
F=\left(T^{3}+600 w_{1} w_{2} w_{3} X Y Z+20\left[3 w_{1} w_{2} w_{3} X Y Z\left(T^{3}+300 w_{1} w_{2} w_{3} X Y Z\right)^{1 / 2}\right]^{1 / 3} .\right.
$$

These equilibrium expressions apply for product positions that satisfy $2\left(w_{2} Y+\right.$ $\left.w_{3} Z\right) \leq p_{2}{ }^{7 A^{*}}-p_{1}{ }^{7 A^{*}}+S<2\left(w_{3} Z+w_{2} Y+w_{1} X\right)$. Whereas the right-hand side is always true, the other left-hand side condition is true if condition (8) fails. This condition, together with $w_{1} X \leq w_{2} Y \leq\left(w_{1} X+w_{2} Y\right) \leq w_{3} Z$, define region $R_{7 \mathrm{~A}}$.

\subsection{PRODUCT EQUILIBRIUM}

We now establish the subgame-perfect equilibrium positions of firms. With subgame perfection, firms anticipate the equilibrium prices in the subgames. We can write profits in the location stage as

$$
\prod_{i}^{\ell}\left(\theta_{1}, \theta_{2}\right) \equiv \Pi_{\mathrm{i}}\left[\mathrm{p}_{1}{ }^{*}\left(\theta_{1}, \theta_{2}\right), \mathrm{p} 2{ }^{*}\left(\theta_{1}, \theta_{2}\right), \theta_{1}, \theta_{2}\right] i=1,2
$$

Thus a change in location has two effects on profits: a direct effect and an indirect effect through prices.

As in two dimensions, we find that maximal differentiation on all attributes, that is, max-max-max is not an equilibrium for any set of attribute weights. The proof is presented in an appendix to an unabridged working paper version of this article available from the authors upon request. In particular, we find that, at the equilibrium locations, the two firms are maximally differentiated on only one attribute and minimally differentiated on the rest. That is, the subgameperfect location-price equilibria are always of the type max-min-min or 
min-max-min or min-min-max. Depending upon the ratio of the weights, there are one, two, or three locational equilibria (and their mirror images).

The first candidate equilibrium is $\left(x_{1}{ }^{*}, y_{1}{ }^{*}, z_{1}{ }^{*}\right)=(1 / 2,1 / 2,0),\left(x_{2}{ }^{*}, y_{2}{ }^{*}, z_{2}{ }^{*}\right)=$ $(1 / 2,1 / 2,1)$, that is, firms are located in the middle of the horizontal planes of the cube, implying minimum differentiation on the $x$ and the $y$ attributes. We call this the min-min-max equilibrium. The second candidate is $\left(x_{1}{ }^{* *}, y_{1}{ }^{* *}, z_{1}{ }^{* *}\right)=$ $(1 / 2,0,1 / 2),\left(x_{2}{ }^{* *}, y_{2}{ }^{* *}, z_{2}{ }^{* *}\right)=(1 / 2,1,1 / 2)$. We call this min-max-min equilibrium. The final candidate equilibrium is given by $\left(x_{1}{ }^{* * *}, y_{1}{ }^{* * * *}, z_{1}{ }^{* * * *}\right)=(0,1 / 2,1 / 2),\left(x_{2}{ }^{* * *}, y_{2}{ }^{* * *}, z_{2}{ }^{* * *}\right)$ $=(1,1 / 2,1 / 2)$. We name this the max-min-min equilibrium. We can show that (a) the min-min-max equilibrium given by $\theta_{1}{ }^{*}=(1 / 2,1 / 2,0), \theta_{2}{ }^{*}=(1 / 2,1 / 2,1)$, holds when the weights satisfy $w_{3} / w_{1} \geq 0.406$ and $w_{3} / w_{2} \geq 0.406$; (b) the min-max-min equilibrium, given by the positions $\theta_{1}^{* *}=(1 / 2,0,1 / 2), \theta_{2}{ }^{* *}=(1 / 2,1,1 / 2)$, holds when $w_{2} / w_{1} \geq 0.406$ and $w_{2} / w_{3} \geq 0.406$; and (c) the max-min-min equilibrium, given by the locations $\theta_{1}^{* * *}=(0,1 / 2,1 / 2), \theta_{2}^{* * * *}=(1,1 / 2,1 / 2)$, holds when $w_{1} / w_{2} \geq 0.406$ and $w_{1} / w_{3}$ $\geq 0.406$.

The method of proof is summarized as follows. Suppose that firm 2 is located at $\left(x_{2}{ }^{*}, y_{2}{ }^{*}, z_{2}{ }^{*}\right)=(1 / 2,1 / 2,1)$. We identify the direction in which profits of firm 1 increase as its location changes by calculating the (vector) gradient of profits $\mathbf{D} \boldsymbol{\Pi}_{1}^{\ell * * *}$. We do this by evaluating analytic expressions for $\mathbf{D} \boldsymbol{\Pi}_{1}^{\ell * * *}$. We identify locations for firm 1 that represent a local maximum, minimum, or saddle point of its profit function. Let $w_{1}<w_{2}$. We find that, when $w_{3}$ is large such that $w_{3} / w_{2}$ $\geq 1$ and $w_{3} / w_{1} \geq 1$, there is only one local maximum of $\boldsymbol{\Pi}_{1}^{\ell * * *}$, at $\boldsymbol{\theta}_{1}=(1 / 2,1 / 2,0)$; therefore it is also a global maximum (in $x_{1}, y_{1}$ and $z_{1}$ ) of the profit function $\mathbf{\Pi}_{1}^{\ell * * * *}$. It follows that for $w_{3} / w_{2} \geq 1$ and $w_{3} / w_{1} \geq 1$, location $(1 / 2,1 / 2,0)$ is the best response to $(1 / 2,1 / 2,1)$. For the same range of weight ratios, by symmetry with respect to the horizontal plane passing through $(1 / 2,1 / 2,1 / 2), \boldsymbol{\theta}_{2}{ }^{*}=(1 / 2,1 / 2,1)$ is the global best response to $(1 / 2,1 / 2,0)$. Thus, for this range of weight ratios, min-min-max is an equilibrium.

Given that firm 2 is located at $\boldsymbol{\theta}_{2}{ }^{*}=(1 / 2,1 / 2,1)$, when $w_{3}$ is smaller so that the weights satisfy $w_{3} / w_{1}>1$ and $0.406<w_{3} / w_{2}<1$, there are two local maxima of firm 1's profits, at $\boldsymbol{\theta}_{1}{ }^{*}=(1 / 2,1 / 2,0)$ and $\boldsymbol{\theta}_{1}{ }^{* *}=(1 / 2,0,1 / 2)$. Let the "middle location" profits of firm 1 be $\Pi_{1}^{\ell}(M) \equiv \Pi_{1}(1 / 2,1 / 2,0)$ and let the "left" profits be $\Pi_{1}^{\ell}(L) \equiv$ $\Pi_{1}(1 / 2,0,1 / 2)$. For the above range of weight ratios, $\Pi_{1}(M)>\Pi_{1}(L)$; therefore $\left(x_{1}{ }^{*}, y_{1}{ }^{*}, z_{1}{ }^{*}\right)=(1 / 2,1 / 2,0)$ is the (global) best reply of firm 1 to $\left(x_{2}{ }^{*}, y_{2}{ }^{*}, z_{2}{ }^{*}\right)=(1 / 2,1 / 2,1)$. By symmetry, $\left(x_{2}{ }^{*}, y_{2}{ }^{*}, z_{2}{ }^{*}\right)=(1 / 2,1 / 2,1)$ is the global best response to $\left(x_{1}{ }^{*}, y_{1}{ }^{*}, z_{1}{ }^{*}\right)=$ $(1 / 2,1 / 2,0)$; therefore min-min-max is a subgame perfect equilibrium.

When $w_{3}$ is reduced further, either (a) $0 \leq w_{3} / w_{2} \leq 0.406$ and $w_{3} / w_{1}>1$, or (b) $0.406<w_{3} / w_{2}<1$ and $0.406<w_{3} / w_{1}<1$ is first satisfied. Consider first the relationships in (a). When the weights satisfy inequalities (a), firm 1's profit function has two local maxima corresponding to the "left" and "middle" locations described above. In contrast to the previous case, in this situation, $\Pi_{1}(M)<\Pi_{1}(L)$; therefore, $\boldsymbol{\theta}_{1}{ }^{* *}=(1 / 2,0,1 / 2)$ is the best reply to $(1 / 2,1 / 2,1)$. However, $\boldsymbol{\theta}_{2}{ }^{*}=(1 / 2,1 / 2,1)$ is not the best reply to $(1 / 2,0,1 / 2)$. This is established as follows. Let Firm 1 be at $(1 / 2,0,1 / 2)$. The problem of the choice of location by firm 2 is symmetric to the 
analogous problem of firm 1 . From the view point of firm 2 , the relative weights are $w_{2} / w_{1}>1$ and $w_{2} / w_{3}>2.463>1$. It follows from previous arguments (made for firm 1$)$ that $(1 / 2,1,1 / 2)$ is a global best reply to $(1 / 2,0,1 / 2)$. Therefore $(1 / 2,1 / 2,1)$ is not the best reply to $(1 / 2,0,1 / 2)$ and min-min-max is not an equilibrium while min-max-min is an equilibrium when inequalities (a) hold.

Consider next the relationhips in (a): $0.406<w_{3} / w_{2}<1$ and $0.406<w_{3} / w_{1}$ $<1$. When inequalities (b) are satisfied, there are three local maxima for firm 1 's profit function. These correspond to the "middle," and "left" locations defined above and a "right" location given by $\boldsymbol{\theta}_{1}{ }^{* * *}=(0,1 / 2,1 / 2)$. Here the best response is the "middle" location $(1 / 2,1 / 2,0)$. Hence, min-min-max is an equilibrium when the weights satisfy conditions (b).

When $w_{3}$ is lowered so that the weights satisfy $0<w_{3} / w_{2} \leq 0.406$ and 0.406 $<w_{3} / w_{1}<1$, there are three local maxima for firm 1's profit function. The global maximum is at the "left" location, that is $(1 / 2,0,1 / 2)$. Once firm 1 is at the "left" location, then as shown above, $(1 / 2,1 / 2,1)$ is no longer the global maximum for firm 2. Firm 2 has an incentive to be at $(1 / 2,1,1 / 2)$ and min-min-max is no longer a subgame-perfect equilibrium. Finally, when $0<w_{3} / w_{2} \leq 0.406$ and $0<w_{3} / w_{1} \leq$ 0.406 , as above, there are three local maxima, but the "left" location for firm 1 is the global maximum, leading to the min-max-min locational equilibrium.

The above argument characterized conditions when min-min-max is an equilibrium. Notice that in the entire discussion above, we assumed $w_{1}<w_{2}$. Hence, under all conditions, the global maxima for firm 1's profits were either at the "left" or the "middle" location. When $w_{2} \leq w_{1}$, we can show that the global maxima would be either at the "right" or the "middle" location.

Finally, by repeating the entire argument described above, but focusing instead on the weight ratios, $w_{2} / w_{1}$ and $w_{2} / w_{3}$, we can characterize the conditions under which min-max-min is an equilibrium. By focusing on $w_{1} / w_{2}$ and $w_{1} / w_{3}$ instead, we can characterize the conditions on the weights for which max-min$\min$ is an equilibrium.

Putting all these together, the regions of existence of these equilibria can easily be illustrated on the three-dimensional simplex in Figure 4, where $w_{1}+$ $w_{2}+w_{3}=1, w_{1}, w_{2}, w_{3} \geq 0$. On segment $A C$ define the points $D$ and $D^{\prime}$ such that $(A D) /(D C)=\left(C D^{\prime}\right) /\left(A D^{\prime}\right)=0.406$, with similar definitions of $E, E^{\prime}, F$, and $F^{\prime}$ on segments $A B$ and $B C$. The region of the weights $\mathbf{w}=\left(w_{1}, w_{2}, w_{3}\right) \in\left(C D H F^{\prime}\right)$ that leads to a min-min-max equilibrium is shaded. Similarly, w $\in\left(A E^{\prime} K D^{\prime}\right)$ leads to a max-min-min equilibrium, and $\mathbf{w} \in(B F M E)$ leads to a min-max-min equilibrium. Notice that, roughly speaking, each equilibrium has maximal differentiation in the dimension that corresponds to the highest weight. Further, when the weights are roughly similar and fall in the central hexagon (MGHIKL), all three equilibria exist. In regions where two weights are high but the third weight is low, two equilibria exist, each with maximal differentiation in the dimension that corresponds one of the two high weights. For example, for $\mathbf{w} \in\left(D G M L D^{\prime}\right)$, min-min-max and max-min-min are the only equilibria. In regions where only one weight is large (close to the vertices) only one equilibrium exists-the one 


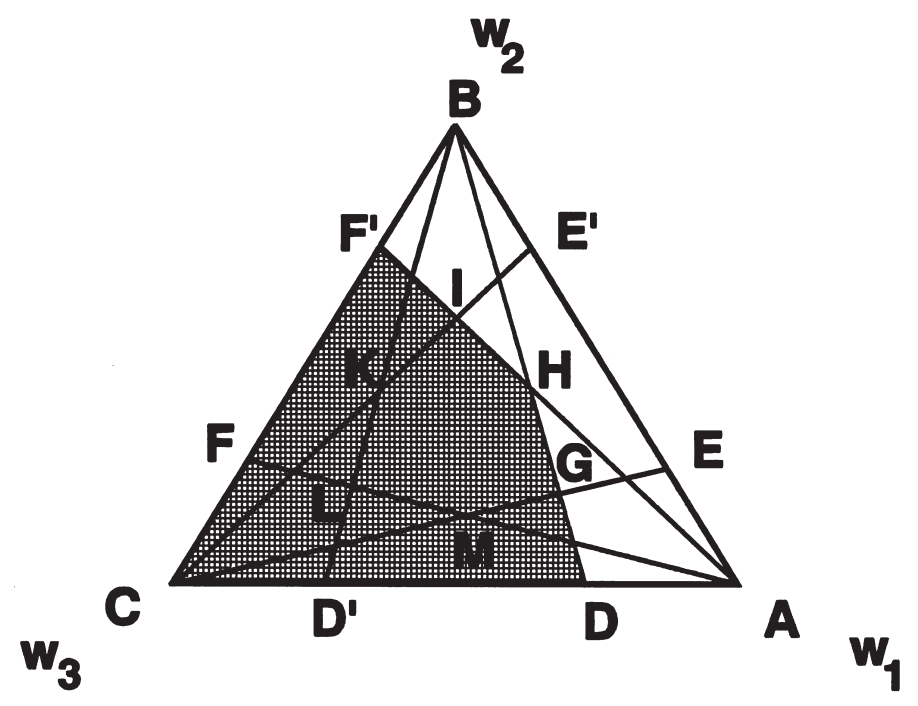

FIGURE 4: Regions Defining Equilibrium Types in the Three-Dimensional Simplex of Preference Weights.

that differentiates maximally in the dimension the large weight. For example, for $\mathbf{w} \in\left(C D^{\prime} L F\right)$ only the max-min-min equilibrium exists.

At each of equilibrium, both firms charge equal prices and share the market equally. This pattern of equilibrium positions confirms our understanding that in multidimensional spaces, firms seek to differentiate their offerings on one dimension only in order to reduce the impact of price competition. Once products are differentiated maximally in one dimension, firms assume identical (central) positions on the other attributes. ${ }^{9}$

\section{CONCLUSION}

In this paper we have examined product positioning and pricing in a multi-attribute framework. We derived subgame-perfect equilibrium positions and associated prices for a duopoly. In one dimension, maximal differentiation holds as shown in D'Aspremont, Jaskold-Gabszewicz and Thisse (1979). We find that, in two dimensions, there are two equilibria when all consumers consider the two attributes as equally important. In each of these equilibria, firms are maximally differentiated on one attribute and minimally differentiated on another. Moreover, when firms are minimally differentiated on one attribute, they occupy central positions on that attribute. We also find that when attributes are differentially weighted by the consumers, so that one attribute has

${ }^{9}$ Finally, we must note that we have not shown that these are the only locational equilibria. However, we were unable to locate any other equilibrium despite extensive search.

(c) Blackwell Publishers 1998 . 
significantly greater importance than the other, only a single equilibrium remains. In this equilibrium firms maximally differentiate on the more important attribute and occupy central positions on the other attribute.

In moving from two to three dimensions we showed that the essential character of the equilibrium does not change. In particular, at the three-dimensional equilibrium, firms are maximally differentiated on one dimension only. In three dimensions, depending on the importance that consumers place on each attribute, there are one, two, or three equilibria. In each equilibrium, firms are maximally differentiated on one attribute and minimally differentiated on the other two. An equilibrium with maximal differentiation in a certain dimension occurs when consumers place sufficient importance on the corresponding attribute. Thus, if consumers place importance only on the first attribute, the equilibrium is max-min-min, that is, it has maximal differentiation in the first dimension only. When consumers also place importance on the second attribute, the min-max-min equilibrium also occurs. Further, when consumers place importance on the third attribute as well, the min-min-max equilibrium occurs in addition to the other two. When all attributes are weighted equally, all three equilibria (max-min-min, min-max-min, and min-min-max) exist.

That the character of the equilibrium is stable is not surprising. Both the two and three-dimensional cases use the preference function defined in Equation (1). In fact, the two-dimensional case can be viewed as a special case of the three-dimensional one where $w_{3}=0$. If only the most important of three attributes is differentiated, then knowledge of the three-dimensional solution implies the two-dimensional and the one-dimensional ones. Reasoning in the reverse direction, if a product is only differentiated on the most important attribute, then we would expect not only the third, but any additional attributes to be minimally differentiated. Unfortunately, we have not yet been able to prove that conjecture.

Up to this point we have used the unit square in two dimensions (and the unit cube in three) as both the space of location of consumers' ideal points and the space of product offerings. Keeping the consumers' space and preference distribution the same, we now allow the space of product offerings to be significantly larger, so that each attribute can range from $1 / 2-k$ to $1 / 2+k$, with $k$ arbitrarily large. The character of equilibrium remains unchanged, with maximal differentiation in only one dimension. In that dimension, products occupy positions outside the consumers' space. The equilibrium locations in two dimensions are $(-1 / 4,1 / 2)$ for firm 1 and $(5 / 4,1 / 2)$ for firm 2 . In three dimensions, for example, the max-min-min equilibrium is at $(-1 / 4,1 / 2,1 / 2)$ and $(5 / 4,1 / 2,1 / 2)$.

An important aspect of our results is the multiplicity of equilibria in both the two-dimensional and the three-dimensional models. When consumers value all attributes roughly equally, all locational $n$-tuples with maximal differentiation in one dimension and minimal differentiation in all others, are equilibria. As more weight is put on a particular dimension, equilibria get eliminated one by one until we reach a unique equilibrium. This shows that advertising can 
have a very important role in eliminating certain equilibria. If advertising can get consumers to pay more attention to a certain product attribute and perhaps weigh it more heavily in preference formation, it can determine which of several equilibria will hold. Therefore, a firm with a unique ability to produce a product consistent with one of the equilibrium positions will certainly want to advertise in an attempt to direct the market to that equilibrium. It is noteworthy that this depends only on the relative importance of the different attributes and not on the relative preferences of individual consumers for different levels of any specific attribute.

There are a number of directions in which these results can be extended. ${ }^{10}$ First, there is the obvious extension to higher-dimensional spaces. Are the equilibrium locations of a $n$-dimensional attribute spaces only differentiated in one dimension? When the weight of one attribute is sufficiently large, the analysis can be confined to case $4 \mathrm{~A}$ of figure 3 . Then our results immediately generalize to $n$-dimensions: the equilibrium is max-min-min-...-min, that is, maximal differentiation in the high weight dimension and minimal differentiation in all other dimensions. This is also observed by Irmen and Thisse (1996). However, the existence and characterization of equilibria when the attribute weights are comparable remain open questions in models with more than three attributes.

Other questions include how do the locational results fare when there are more than two competitors and for what classes of distributions can we extend our duopoly-positioning results. All these are very interesting questions that we leave for further research.

\section{REFERENCES}

Anderson, Simon P., Andre de Palma, and Jacques-François Thisse. 1993. Discrete Models of Product Differentiation. Cambridge, MA: MIT Press.

Ansari, Asim, Nicholas Economides, and Avijit Ghosh. 1994. "The Defender Model with Nonuniform Preferences," Marketing Science, 13, 248-273.

Ben-Alsiva, M., André de Palma, and Jacques-François Thisse. 1989. "Spatial Competition with Differentiated Products,” Journal of Regional Science and Urban Economics, 19, 5-19.

\footnotetext{
${ }^{10}$ It should be clear that the principle of Max-Min-Min differentiation is dependent on the assumptions implicit in our framework. As these assumptions are relaxed the character of the equilibrium may indeed change. For example, in a two-dimensional spatial model with one dimension providing linear disutility and the other providing quadratic, Ben-Akiva, De Palma, and Thisse (1989) show that the likelihood of minimal differentiation in the second dimension varies as a function of the absolute values of the weights of the utility function, the size of the market, the degree of heterogeneity in the market, and the number of firms. Some of these characteristics are fixed in our model. In particular, we only allow two firms. Additionally, the size of the market and the degree of heterogeneity are fixed in our model by the assumptions that the product space is a unit square (in two dimensions) or a unit cube (dimension three) and that consumers are uniformly distributed over it. While the Ben-Akiva, DePalma, and Thisse (1989) results come from a different model than ours, they suggest that the max-min and the max-min-min results might vanish if the product space or other aspects of the problem were altered. These are questions for future research.
}

(c) Blackwell Publishers 1998. 
Caplin, Andrew, and Barry Nalebuff. 1991. "Aggregation and Imperfect Competition: On the Existence of Equilibrium," Econometrica, 59, 25-59.

D’Aspremont, Claude, Jean Jaskold-Gabszewicz, and Jacques-François Thisse. 1979. "On Hotelling's 'Stability in Competition', Econometrica, 47, 1145-1150.

Economides, Nicholas. 1984. "The Principle of Minimum Differentiation Revisited," European Economic Review, 24, 345-368.

—. 1986a. "Nash Equilibrium Existence in Duopoly with Products Defined by Two Characteristics," Rand Journal of Economics, 17, 431-439.

_. 1986b. "Minimal and Maximal Product Differentiation in Hotelling's Duopoly," Economics Letters, 21, 67-71.

.1989. "Symmetric Equilibrium Existence and Optimality in Differentiated Products Markets,” Journal of Economic Theory, 47, 178-194.

—. 1993. "Quality Variations in the Circular Model of Variety Differentiated Products," Regional Science and Urban Economics, 23, 235-257.

Green, Paul E. and V. Srinivasan. 1978. "Conjoint Analysis in Consumer Research: Issues and Outlook," Journal of Consumer Research, 5, 103-123.

Hauser, John R. 1988. "Competitive Price and Positioning Strategies," Marketing Science, 7, 76-91.

Hauser, John R., and M. Shugan. 1983. "Defensive Marketing Strategy," Marketing Science, 2, 319-360.

Hotelling, Harold. 1929. "Stability in Competition," Economic Journal, 39, 41-57.

Irmen, Andreas, and Jacques-Francois Thisse. 1996. "Competition in Multi-Characteristics Spaces: Hotelling was Almost Right,” Discussion Paper No. 1446, Center for Economic Policy Research, London, October.

Kumar, R. V., and D. Sudarshan. 1988. "Defensive Marketing Strategies: An Equilibrium Analysis Based on Decoupled Response Function Models," Management Science, 34, 805-815.

Moorthy, Sridhar K. 1986. "Product and Price Competition in a Duopoly," Marketing Science, 5, 141-168.

Neven, Damien. 1985. “Two-stage (Perfect) Equilibrium in Hotelling's Model,” Journal of Industrial Economics, 33, 317-326.

Neven, Damien, and Jacques-François Thisse. 1990. "On Quality and Variety Competition,” J.J. Gabszewicz, J.-F. Richard and L.A. Wolsey (eds.), in Economic Decision-Making: Games, Econometrics and Optimization, Amsterdam: North-Holland, pp. 175-199.

Rao, Vithala R. and Joel H. Steckel. 1995. "A Cross-Cultural Analysis of Price Response to Environmental Changes," Marketing Letters, 6, 1-12.

Salop, Steven C. 1979b. "Monopolistic Competition with Outside Goods," Bell Journal of Economics, 10, 141-156.

Tabuchi, Takatoshi. 1994. "Two-Stage Two Dimensional Spatial Competition between Two Firms," Regional Science and Urban Economics, 24, 207-227.

Vandenbosch, Mark B., and Charles B. Weinberg. 1995. "Product and Price Competition in a Two-Dimensional Vertical Differentiation Model," Marketing Science.

Vickrey, William S. 1964. Microstatics. New York: Harcourt, Brace and World.

\section{APPENDIX}

\section{DEMAND DEFINITIONS FOR THE THREE-DIMENSIONAL MODEL}

We first describe the demand expressions and regions pertaining to Scenario $1 \mathrm{~B}$, and then describe the relationships among the remaining scenarios.

\section{Scenario $1 B$}

Case $1 B$. When $0 \leq L \leq 2 w_{1} X$, demand for firm 1 is $D_{1}{ }^{1 \mathrm{~B}}=D_{1}{ }^{1 \mathrm{~A}}$;

Case $2 B$. When $2 w_{1} X \leq L \leq 2 w_{2} Y$, demand for firm 1 is $D_{1}{ }^{2 \mathrm{~B}}=D_{1}{ }^{2 \mathrm{~A}}$; 
Case $3 B$. When $2 w_{2} Y \leq L \leq 2 w_{3} Z$, demand is $D_{1}^{3 \mathrm{~B}}=D_{1}{ }^{3 \mathrm{~A}}$;

Case $4 B$. When $\left.2 w_{3} Z \leq L \leq 2\left(w_{1} X+w_{2} Y\right), D_{1}{ }^{4 \mathrm{~B}}=D_{1}{ }^{3 \mathrm{~A}}-\left(L-2 w_{3} Z\right)\right)^{3} / H$;

Case $5 B$. When $2\left(w_{1} X+w_{2} Y\right) \leq L \leq 2\left(w_{1} X+w_{3} Z\right), D_{1}{ }^{5 \mathrm{~B}}=D_{1}^{5 \mathrm{~A}}$;

Case 6B. When $2\left(w_{1} X+w_{3} Z\right) \leq L \leq 2\left(w_{2} Y+w_{3} Z\right)$, we have $D_{1}^{6 \mathrm{~B}}=\mathrm{D}_{1}{ }^{6 \mathrm{~A}}$; and

Case $7 B$. When $2\left(w_{2} Y+w_{3} Z\right) \leq L \leq 2\left(w_{1} X+w_{2} Y+w_{3} Z\right), D_{1}{ }^{7 \mathrm{~B}}=\mathrm{D}_{1}{ }^{7 \mathrm{~A}}$.

As is evident above, the two scenarios differ across only one demand expression. However, the regions of the product space associated with Cases 3 , 4 , and 5, are different across the two scenarios.

We now show how the remaining scenarios can be obtained from the two that were analyzed above. We define transformation rules that we use on the above derived demand expressions and price inequalities so as to obtain the corresponding expressions in the other scenarios. The transformation rules are as follows:

$$
\begin{gathered}
\operatorname{tran} 2=\left(w_{1} X \rightarrow w_{1} X, w_{2} Y \rightarrow w_{3} Z, w_{3} Z \rightarrow w_{2} Y\right) ; \\
\operatorname{tran} 3=\left(w_{1} X \rightarrow w_{2} Y, w_{2} Y \rightarrow w_{1} X, w_{3} Z \rightarrow w_{3} Z\right) ; \\
\operatorname{tran} 4=\left(w_{1} X \rightarrow w_{2} Y, w_{2} Y \rightarrow w_{3} Z, w_{3} Z \rightarrow w_{1} X\right) ; \\
\operatorname{tran} 5=\left(w_{1} X \rightarrow w_{3} Z, w_{2} Y \rightarrow w_{1} X, w_{3} Z \rightarrow w_{2} Y\right) ; \text { and } \\
\operatorname{tran} 6=\left(w_{1} X \rightarrow w_{3} Z, w_{2} Y \rightarrow w_{2} Y, w_{3} Z \rightarrow w_{1} X\right) .
\end{gathered}
$$

These rules work as follows. In order to obtain the seven cases of demand and the associated price domains for Scenario $2 \mathrm{~A}$, we apply tran2 on the corresponding demand expressions and price domains of Scenario 1A. For example, the demand expression for Case 1 of Scenario $2 \mathrm{~A}$ can be obtained by simultaneously substituting in $D_{1}^{1 \mathrm{~A}}$ above, $w_{1} X$ in place of $w_{1} X, w_{3} Z$ in place of $w_{2} Y$, and $w_{2} Y$ in place of $w_{3} Z$. These replacement rules follow from the geometric symmetry associated with the sides of the unit cube. Similarly, Scenario 2B can be analyzed by applying tran 2 on the corresponding expressions of Scenario 1B. The other scenarios can be analyzed in an analogous manner. 